\title{
Automated phenotyping of plant shoots using imaging methods for analysis of plant stress responses - a review
}

\author{
Jan F Humplík ${ }^{1 \dagger}$, Dušan Lazár ${ }^{2 \dagger}$, Alexandra Husičková ${ }^{2}$ and Lukáš Spíchal ${ }^{1 *}$
}

\begin{abstract}
Current methods of in-house plant phenotyping are providing a powerful new tool for plant biology studies. The self-constructed and commercial platforms established in the last few years, employ non-destructive methods and measurements on a large and high-throughput scale. The platforms offer to certain extent, automated measurements, using either simple single sensor analysis, or advanced integrative simultaneous analysis by multiple sensors. However, due to the complexity of the approaches used, it is not always clear what such forms of plant phenotyping can offer the potential end-user, i.e. plant biologist. This review focuses on imaging methods used in the phenotyping of plant shoots including a brief survey of the sensors used. To open up this topic to a broader audience, we provide here a simple introduction to the principles of automated non-destructive analysis, namely RGB, chlorophyll fluorescence, thermal and hyperspectral imaging. We further on present an overview on how and to which extent, the automated integrative in-house phenotyping platforms have been used recently to study the responses of plants to various changing environments.
\end{abstract}

Keywords: Plant phenotyping, RGB digital imaging, Chlorophyll fluorescence imaging, Thermal imaging, Hyperspectral imaging, Shoot growth, Biomass production

\section{Introduction}

Recently, a large number of reviews have been published on the advantages and possibilities of high-throughput plant phenotyping approaches [1-5]. Most focus on the potential of these approaches which use precise and sophisticated tools and methodologies to study plant growth and development. To review the state-of-the-art of phenotyping platforms, we present a list of recent publications in Table 1 . Interestingly, in about a half of these, only one measuring tool, mostly RGB imaging, for plant phenotyping was used. In the other papers, integrative phenotyping, signifying two or more measuring tools but which are rarely automated, was used (Table 1). This illustrates that the integrative automated highthroughput phenotyping measurements/platforms are still rather rare. Greenhouse- and grow chamber-based

\footnotetext{
* Correspondence: lukas.spichal@upol.cz

${ }^{\dagger}$ Equal contributors

'Department of Chemical Biology and Genetics, Centre of the Region Haná for Biotechnological and Agricultural Research, Faculty of Science, Palacký

University, Šlechtitelů 11, Olomouc CZ-78371, Czech Republic

Full list of author information is available at the end of the article
}

plant phenotyping platforms are publically available and these offer their services and collaborative projects. Descriptions, methodological background and focus can be found at http://www.plant-phenotyping-network.eu/eppn/ select_installation. As an example of the integrative automated high-throughput phenotyping platform, a grow chamber-based phenotyping facility installed at Palacký University in Olomouc, Czech Republic is presented in Figure 1.

High-throughput integrative phenotyping facilities provide an opportunity to combine various methods of automated, simultaneous, non-destructive analyses of plant growth, morphology and physiology, providing a complex picture of the plant growth and vigour in one run, and repeatedly during the plant's life-span. Particular methods used in integrative plant phenotyping are often not new and usually represent those which have already been used for a number of years in basic research, e.g. noninvasive methods that employ visible or fluorescence imaging (described in more detail further in the text). High-throughput then allows analysis of the plants on a 
Table 1 List of selected works describing automated high-throughput analysis to study plant stress responses

\begin{tabular}{|c|c|c|c|c|c|}
\hline Study & Plant species & Type of stress & Type of the study & Type of automated analysis & $\begin{array}{l}\text { Platform } \\
\text { name/origin }\end{array}$ \\
\hline Granier et al. 2006; [58] & Arabidopsis & drought-stress & methodology & RGB (top view) & PHENOPSIS \\
\hline Skirycz et al. 2011; [59] & Arabidopsis & drought-stress & applied & RGB (top view) & WIWAM \\
\hline Clauw et al. 2015; [60] & Arabidopsis & drought-stress & applied & RGB (top view) & WIWAM \\
\hline Tisné et al. 2013; [61] & Arabidopsis & drought-stress & applied & RGB (top view) & PHENOSCOPE \\
\hline Neumann et al. 2015; [26] & barley & drought-stress & methodology & RGB (multiple views) & LemnaTec \\
\hline Pereyra-Irujo et al. 2012; [62] & soybean & drought-stress & methodology & RGB (two-views) & $\begin{array}{l}\text { GlyPh (self- } \\
\text { construction) }\end{array}$ \\
\hline Honsdorf et al. 2014; [16] & barley, (wild species) & drought-stress & applied & RGB (multiple views) & LemnaTec \\
\hline Coupel-Ledru et al. 2014; [63] & grapevine & drought-stress & applied & RGB (multiple views) & LemnaTec \\
\hline Petrozza et al. 2014; [66] & tomato & drought-stress & applied & $\begin{array}{l}\text { RGB (multiple views), } \\
\text { hyperspectral NIR, SLCFIM }\end{array}$ & LemnaTec \\
\hline Harshavardhan et al. 2014; [67] & Arabidopsis & drought-stress & applied & $\begin{array}{l}\text { RGB (top view), } \\
\text { hyperspectral NIR }\end{array}$ & LemnaTec \\
\hline Bresson et al. 2013; [68] & Arabidopsis & drought-stress & applied & RGB (top view) & PHENOPSIS \\
\hline Bresson et al. 2014; [69] & Arabidopsis & drought-stress & applied & RGB (top view), TLCFIM & PHENOPSIS \\
\hline Chen et al. 2014; [64] & barley & drought-stress & methodology & $\begin{array}{l}\text { RGB (multiple-views), } \\
\text { hyperspectral NIR, SLCFIM }\end{array}$ & LemnaTec \\
\hline Fehér-Juhász et al. 2014; [19] & wheat & drought-stress & applied & $\begin{array}{l}\text { RGB (multiple views), } \\
\text { thermoimaging }\end{array}$ & $\begin{array}{l}\text { self-construction, } \\
\text { semi-automated }\end{array}$ \\
\hline Cseri et al. 2013; [65] & barley & drought-stress & methodology & $\begin{array}{l}\text { RGB (multiple views), } \\
\text { thermoimaging }\end{array}$ & $\begin{array}{l}\text { self-construction, } \\
\text { semi-automated }\end{array}$ \\
\hline Vasseur et al. 2014 [71] & Arabidopsis & $\begin{array}{l}\text { heat-stress, } \\
\text { drought-stress }\end{array}$ & applied & RGB (top view) & PHENOPSIS \\
\hline Rajendran et al. 2009; [73] & wheat & salt-stress & applied & RGB (multiple views) & LemnaTec \\
\hline Harris et al. 2010; [74] & wheat, barley & salt-stress & applied & RGB (multiple views) & LemnaTec \\
\hline Golzarian et al. 2011; [18] & barley & salt-stress & methodology & RGB (multiple views) & LemnaTec \\
\hline Schilling et al. 2014; [75] & barley & salt-stress & applied & RGB (multiple views) & LemnaTec \\
\hline Hairmansis et al. 2014; [76] & rice & salt-stress & applied & RGB (multiple views) SLCFIM & LemnaTec \\
\hline Chaerle et al. 2006; [77] & tobacco & biotic-stress & methodology & thermoimaging, TLCFIM & self-construction \\
\hline Poiré et al. 2014; [79] & Brachypodium & nutrient-deficiency & methodology & RGB (multiple views ) & LemnaTec \\
\hline Neilson et al. 2015; [80] & Sorghum & nutrient-deficiency & methodology & $\begin{array}{l}\text { RGB (multiple views), } \\
\text { hyperspectral NIR }\end{array}$ & LemnaTec \\
\hline Chaerle et al. 2007; [81] & bean & $\begin{array}{l}\text { nutrient-deficiency, } \\
\text { biotic-stress }\end{array}$ & methodology & $\begin{array}{l}\text { RGB (top view), } \\
\text { thermoimaging, TLCFIM }\end{array}$ & self-construction \\
\hline Jansen et al. 2009; [37] & Arabidopsis, tobacco & $\begin{array}{l}\text { drought-stress, } \\
\text { chilling-stress }\end{array}$ & methodology & RGB (top view), KCFIM & $\begin{array}{l}\text { GROWSCREEN } \\
\text { (self-construction) }\end{array}$ \\
\hline Humplík et al. 2015; [20] & pea, field cultivars & cold-stress & methodology & RGB (multiple views), KCFIM & PlantScreen \\
\hline
\end{tabular}

large scale. This enables users to apply statistics to discover subtle but significant differences between the studied genotypes and treatment variants.

The potential users of such facilities, mostly biologists, are often not very familiar with the applied physical methods used in integrative plant phenotyping. Thus, in this mini-review, we present a simple introduction to the basis of various non-invasive sensors used in highthroughput phenotyping platforms, namely visible redgreen-blue (RGB) imaging, chlorophyll fluorescence imaging (CFIM), thermoimaging, and hyperspectral imaging. Further, we describe potential applications of some of the phenotyping methods that have been used to study the responses of different plant species to various stresses.

\section{Non-destructive analysis of growth and physiology of plant shoots}

The methods for automated phenotyping and their aims have been reviewed in a number of recent reports $[3,6,7]$. In the following text we give a description of the basis of the automated non-invasive analysis of plant 


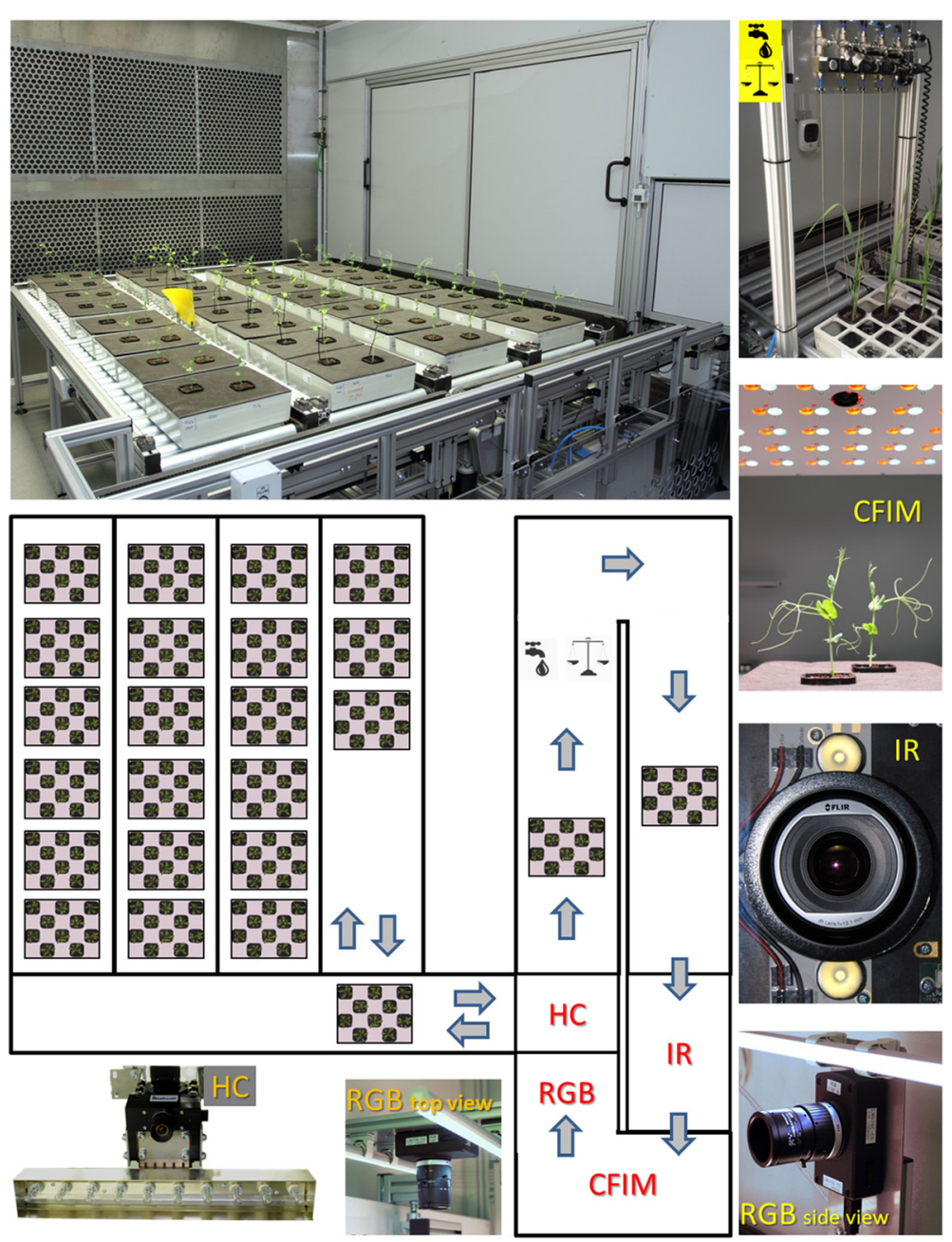

Figure 1 Scheme of the grow chamber-based automated high-throughput phenotyping platform PlantScreen ${ }^{\text {TM }}$ (Photons Systems Instruments, Brno, Czech Republic), installed at Palacký University in Olomouc, Czech Republic [20]. The system is located in a growth chamber with white LED illumination (max. $1000 \mu \mathrm{mol}$ photons $\mathrm{m}^{-2} \mathrm{~s}^{-1}$ ) and controlled environment $\left(10-40^{\circ} \mathrm{C}, 30-99 \%\right.$ relative humidity). The growth area with roller conveyer has capacity of up to 640 Arabidopsis, cereals and other crops grown in standardized pots. The measuring cabinet contains acclimation chamber for dark adaptation of plants coupled with an automated weighting and watering area. The cabinet is equipped with KCFIM and RGB imaging (top and 2 side views), thermoimaging (IR) to measure stomata openness and SWIR hyperspectral imaging to determine water content. The platform can be controlled either from the place or via remote control software. The operating software enables automatic data evaluation.

shoots and appropriate sensors that have been used for studies of plant stress responses.

\section{Visible RGB imaging of plant shoots}

Apart from the importance of root-growth analysis, a key descriptive parameter in plant physiology is the growth of plant shoots. Although there are numerous secondary traits describing the morphology of shoots in particular species and their developmental stages, the primary and universal trait is biomass formation. Shoot biomass is defined as the total mass of all the aboveground plant parts at a given point in a plant's life [8]. This trait can be easily assessed by a simple weighing of the fresh (FW) and dry (DW) masses. However, this involves the destruction of the measured plant thus only allowing end-point analyses. Similarly, leaf area and consequently the plant growth rate are usually determined by manual measurements of the dimensions of plant leaves [9-11]. Such measurements are highly time consuming and thus cannot be used for large scale experiments. For this reason, plant phenotyping facilities prefer to evaluate the growth rate using imaging methods which employ digital cameras with subsequent software image analysis. This enables a faster and more precise determination of the leaf area [12-14] and other parameters called projected area (Figure 2), or hull area in the case of monocots $[15,16]$. In general, non-invasive techniques of shoot growth determination have proven very reliable, and high correlations between the digital area and the shoot fresh, or dry weights, respectively, 


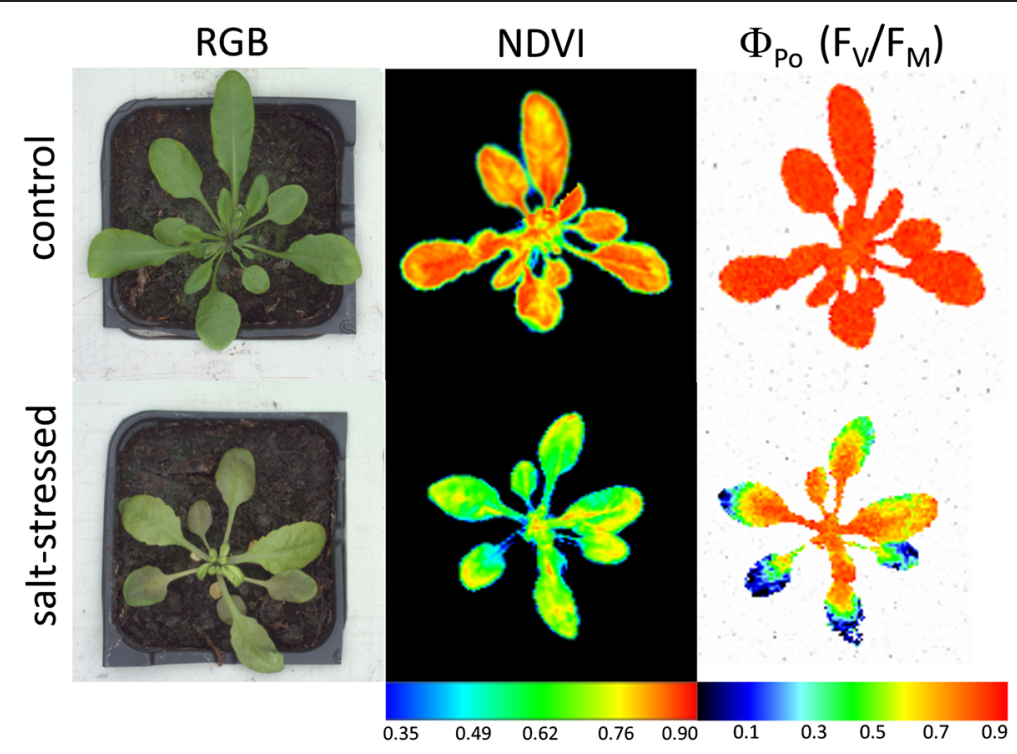

Figure 2 The illustrative figure presenting outcome of simultaneous analysis of control and salt-stressed Arabidopsis plants, using RGB, hyperspectral and Chl fluorescence imaging. The 18 DAG old soil-grown Arabidospis plants were treated with $250 \mathrm{mM} \mathrm{NaCl}$ (salt-stressed) and water (control) and after 48 hours were analysed by different sensors for comparison in: morphology (top-view RGB imaging can be used for computation of rosette area or shape parameters), spatial distribution of vegetation index reflecting changes in the chlorophyll content (NDVI) provided by VIS/NIR hyperspectral camera, and the changes in maximal quantum yield of PSII photochemistry for a dark-adapted state ( $\Phi_{\mathrm{Po}}$, also referred as $\mathrm{F}_{\mathrm{V}} / \mathrm{F}_{\mathrm{M}}$ ) reflecting the photosynthetic activity of the plants obtained from KCFIM.

were reported in Arabidopsis, tobacco [17], cereals $[18,19]$, and pea [20]. An example of a general shoot phenotyping protocol based on biomass estimation was reported by Berger et al. [21]. Similarly, other common morphometric parameters such as stem length, number of tillers and inflorescence architecture can be assessed non-destructively and manually, but again the time requirements, limit the number of plants analysed. Highthroughput approaches for analyses of these rather species-specific traits would be very valuable [15], however, with the exception of Arabidopsis [22] the range of accessible solutions is still limited (for some emerging methods see [23-26]).

Correct determination of digital plant growth area can be distorted by overlapping leaves, leaf twisting and curling, and circadian movement, especially when the RGB image is taken only from one view (e.g. from top view). A new approach developed for Arabidopsis consisting of plant area estimation (which takes into account leaf overlapping), growth modelling and analysis, followed by application of a nonlinear growth model to generate growth curves, and subsequent functional data analysis, was shown to analyse the plant growth in highthroughput experiments more precisely [14]. However, due to the use of only a top-view RGB imaging, this approach cannot be applied for analyses of most of the agronomical important plants with vertical growth. A set-up that introduces more projections (e.g. side-views) into the phenotyping platforms thus can partially solve this problem. The three-views RGB imaging together with linear mathematical modelling was used for accurate estimation of plant shoot dry weight of wheat and barley from two dimensional images [18]. The accuracy of three-view approach has been recently validated in species with challenging shoot morphology such as field pea [20].

\section{Chlorophyll fluorescence imaging (CFIM)}

One of the chlorophyll (Chl) fluorescence methods is chlorophyll fluorescence induction (CFIN), i.e., the measurement of the Chl fluorescence signal during illumination of the sample following prior dark adaptation. Since the first paper on CFIN by Kautsky and Hirsch [27], CFIN has been one of the most common methods used in photosynthesis and plant physiology research: it is inexpensive, non-destructive, and above all, provides a great deal of information about the photosynthetic function of the sample (reviewed, e.g., by Lazár [28,29]). Use of pulse amplitude modulation (PAM) techniques for the measurement of CFIN together with the application of the saturation pulse (SP) method enables the separation of photochemical and non-photochemical events occurring in the sample [30]. Chl fluorescence is excited and measured with the help of weak measuring flashes, whereas photosynthesis is maintained by actinic illumination and saturation of photosynthesis is achieved by the SPs. Since Chls absorb in blue (Chl $a$ at $436 \mathrm{~nm}$ and $\mathrm{Chl}$ $b$ at $470 \mathrm{~nm}$, respectively) and red (at about $650 \mathrm{~nm}$ for 
both Chls $a$ and $b$ ) regions of visible spectrum, the measuring and actinic light is the light with one of the above wavelengths, usually $650-\mathrm{nm}$. The SPs are usually generated by white light. On the other hand, Chl fluorescence emission spectrum at room temperature shows two peaks centred at about 680 and $735 \mathrm{~nm}$. To avoid a possible overlap of the 650-nm excitation light with $\mathrm{Chl}$ fluorescence emission, the Chl fluorescence signal is detected at wavelengths longer than $700 \mathrm{~nm}$. To reveal spatial heterogeneity of the fluorescence signal during CFIN, imaging Chl fluorometers were developed [31,32]. In the images (for illustration see Figure 2), different colours are used to show different fluorescence intensities according to a chosen false colour scale (as mentioned above, fluorescence emission is always above $700 \mathrm{~nm}$, red light). An additional advantage of the CFIM is that it provides a huge amount of data which can be thoroughly analysed and used for early detection of plant stress as shown, e.g., by Lazár et al. [33]. At present, modern CFIM instruments adopt PAM and SP methods/techniques and are thus highly suitable for high-throughput plant phenotyping (reviewed, e.g., by Gorbe and Calatayud [34], Harbinson et al. [35]). However, over the course of time, too many Chl fluorescence parameters were defined and claimed to reflect particular functions of photosynthetic apparatus. Hence, there is a problem over which parameter should be measured/evaluated and presented. Values of most of the parameters cannot be mutually compared. It is only possible to compare relative changes (caused, e.g., by a stress treatment) of a given parameter. The parameters of the so-called energy partitioning, i.e., quantum yields of processes responsible for the use of the absorbed light energy, are the best choice (reviewed by Lazár [36]) as they are all defined on the same basis and can be directly compared. Since all quantum yields sum to unity, the quantum yields express fractions of absorbed excitation light that are used for given processes (photochemical and various types of non-photochemical energy dissipations).

It is also worth mentioning here that kinetic types of CFIM (KCFIM) that measure whole CFIN and also apply the SPs which then allow computation of various $\mathrm{Chl}$ fluorescence parameters, and integrate signal from the whole leaf or shoot, are the most valuable for physiological studies. However, integration of KCFIM into high-throughput systems [20,37] is not very common and in the majority of recent reports, imaging systems measuring either single Chl fluorescence level (SLCFIM) or two Chl fluorescence levels (usually the minimal and maximal Chl fluorescence levels for the dark-adapted state; TLCFIM) were used (see Table 1). As intensity of Chl fluorescence depends on the amount of chlorophylls, the SLCFIM might be used, e.g. to distinguish between non-stressed and senescent leaves (when the amount of Chls is decreased) at the later stages of stress progression but it does not provide any information about early processes in photosytem II (PSII) that are not necessarily linked to the later senescence events. Further, the usual output of the TLCFIM, the $\mathrm{F}_{\mathrm{V}} / \mathrm{F}_{\mathrm{M}}$ ratio, which estimates the maximum quantum yield of photosystem II photochemistry, provides only a limited information about photosynthetic function compared with the outputs of the KCFIMs, which also allow determination of the other quantum yields and parameters (see [36] for a review).

\section{Thermoimaging}

Plants are cooled by transpiration and when the stomata are closed, plant temperature increases. Based on this principle, thermal imaging was used for the first time to detect the changes in the temperature of sunflower leaves caused by water deficiency [38]. In addition to transpiration, stomata also drive water vapour, both parameters being typically determined by leaf gas exchange measurements. However, leaf gasometry involves contact with leaves which often interferes with their function. Further, leaf gasometry is time-consuming, limited by sample size and/or large number of samples required. In addition to heat emission, plants can lose heat by conduction and convection, which in fact represent mechanisms of a non-photochemical quenching of excited states. For this reason, it is not unexpected that an increased thermal signal correlates with an increase in non-photochemical quenching as shown by Kaňa and Vass [39]. Given the foregoing, thermoimaging is a very suitable method for plant phenotyping [19,40,41]. Like CFIM, it uses cameras to measure spatial heterogeneity of heat emissions, usually from leaves; the heat is electromagnetic radiation in the infrared region, usually between $8-13 \mu \mathrm{m}$. Generally, thermal imaging has been successfully used in a wide range of conditions and with diverse plant species. The technique can be applied to different scales, e.g., from single seedlings/leaves through whole trees or field crops to regions. However, researchers have to keep in mind that environmental variability, e.g., in light intensity, temperature, relative humidity, wind speed, etc. affects the accuracy of thermal imaging measurements and therefore the measurements and their interpretations must be done with care. Although thermal imaging sensors have been integrated into the in-house phenotyping platforms with controlled-environment (see section The use of phenotyping methods to study plant stress responses) the majority of studies have been performed so far in field conditions [42-44]. All aspects of thermal imaging used for the exploration of plant-environment interactions, as well as an overview of the application of thermoimaging in field phenotyping, were recently reviewed by Costa et al. [45]. 


\section{Hyperspectral imaging (VIS-NIR, SWIR)}

The absorption of light by endogenous plant compounds is used for calculations of many indices which reflect the composition and function of a plant. Such indices are, for example, the normalized difference vegetation index (NDVI) [46], an estimator of the Chl content, and the photochemical reflectance index (PRI) [47], an estimator of the photosynthetic efficiency. The absorption of a compound (e.g., water) at a given wavelength [48] can also be used for direct estimation of the compound contents in the plant. For practical reasons, measurement of absorbance is replaced here by measurements of reflectance. Depending on the measured wavelengths of reflected signal, various detectors are used, usually VIS-NIR (visible-near infrared region (400-750) - (750-1400 nm)) and SWIR (short wavelength infrared region; 1400$3000 \mathrm{~nm})$. Measurements of the reflectance signal in VIS-NIR and SWIR regions originate from methods of remote sensing [49-51]. However, due to the high value of the information they carry, they are very suitable methods for plant phenotyping [52-54]. The reflectance signal can be detected at selected wavelengths or separated spectral bands (so-called multispectral detection). The whole spectral region can also be measured even for each pixel when cameras are applied and the hyperspectral imaging is carried out (Figure 2). Whereas the hyperspectral imaging in the VIS-NIR spectral region is used for evaluation of several indices as mentioned above, the SWIR spectral region is mainly used for the estimation of the plant's water content. Several aspects of plant reflectance were recently reviewed by Ollinger [55]. Despite the many indices that have been defined so far, based on the reflectance measurements, it is difficult to assess them accurately, similar to the situation with CFIN parameters (see above). For this reason, critical revision of all of the reflectance indices is needed to evaluate which of them provide the required information in the best way.

\section{The use of phenotyping methods to study plant stress responses}

One of the most important applications of automated plant phenotyping methods is in studies of plants' responses to various types of environmental stresses. In Table 1 we listed recent reports describing phenotyping protocols developed for indoor automated shoot phenotyping used in stress-related studies. Since the integrative approaches are a logical but rather new step in the development of phenotyping platforms, there are limited reports on the use of simultaneous analysis by multiple sensors. For this reason, we included here "singlesensor" experiments as well, that were performed in the automated platforms.
Perhaps the most widely used application of highthroughput phenotyping is in the search for droughttolerant varieties. Objectives, traits and approaches related to automated plant selection for drought stress resistance were recently reviewed in Mir et al. [56], and Berger et al. [57]. Here, we add information from examples of the use of non-invasive plant phenotyping in this field. One of the early reports on the use of the highthroughput phenotyping platform describes the employment of the commercial-prototype system for evaluation of drought tolerance in nine Arabidopsis accessions [58]. The screening was based on RGB imaging, estimating rosette-leaf area and automated pot weighing and watering to assess transpiration rates. A very similar approach was later used by Skirycz et al. also in Arabidopsis [59]. The same platform was further used in a recent physiological study of Clauw and co-authors in which the impact of mild-drought on various Arabidopsis thaliana accessions was evaluated [60]. Another study on Arabidopsis employing top-view RGB imaging, pot weighing and automated rotation of pots was performed by Tisné et al. [61]. The phenotyping platform was designed to prevent position effect on water evaporation and authors demonstrated important improvement in the evaporation homogeneity [61].

Although these studies represent an important contribution to the development of automated phenotyping, the design of the platform for top-view experiments has limited their use to analyses of plants with leaf rosette. Further progress thus lay in development of platforms allowing RGB imaging from multiple positions. The most recent advances in the use of multiple-view RGB imaging followed by software analysis were demonstrated in a study by Neumann et al. [26]. The authors were able to automatically extract from the images of the barley plants, the plant height and width, and also leaf colours to evaluate the impact of drought on the degradation of chlorophyll. Earlier, Pereyra-Irujo et al. [62], reported a study that employed a self-constructed high-throughput platform for the RGB screening of growth and water-use efficiency (WUE) in two soybean (Glycine max L.) genotypes. The system with automated weighing and watering placed in the greenhouse was used to analyse the projected area of the shoots and the mass of the pots [62]. An impressive number of plants was analysed for similar traits in the study by Honsdorf et al. [16]. These authors searched for drought-tolerance QTLs in 48 wild barley introgression lines, using a commercial greenhouse based platform with multiple-view RGB imaging and automated weighing and watering [16]. A similar approach utilizing estimation of shoot biomass based on RGB imaging was used by CoupelLedru et al., to screen thousands of grapevine plants for drought tolerance [63]. In these studies, the plant water 
management was automatically analysed by simple weighing of the pots. This approach, however, begs several questions about the homogeneity of evaporation from the soil of the pots placed in different positions of the growing area. The solution to this issue usually requires an exhaustive validation process with numerous control pots and artificial plant-like objects randomly distributed throughout the growing area (Mark Tester, personal communication). A more elegant solution could be the use of the specific sensors controlling directly the plant water content [64] or transpiration [65] of each plant. Even this approach, however, requires appropriate validation.

An integrative way of analysis was employed in the study of Petrozza et al. [66]. Here, the effect of Megafol treatment on drought-stressed tomatoes was assessed using RGB imaging to distinguish shoot area, SLCFIM measurement to calculate "stress index" and NIR camera for water content estimation. Repeated measurements by NIR camera throughout the experiment allowed visualizing the drop of the high water content index that precedes the growth limitation caused by drought stress [66]. A combination of RGB and NIR imaging techniques was also used by Harshavardhan et al. for analysis of the drought-tolerance of transgenic Arabidopsis plants [67]. The RGB imaging was employed by Bresson et al. to study the effect of plant-bacteria interactions on plant tolerance to drought stress [68]. The integration of $F_{V} / F_{M}$ measurement by TLCFIM provided complementary information to the growth rate and WUE analysis obtained by pot weighing [69]. A combination of RGB, SLCFIM and NIR imaging techniques was used by Chen et al. [64] to study different phenotypic traits of 18 barley genotypes. The authors used sophisticated statistics and mathematical modelling to classify genotypes based on their response to drought stress [64].

Another important trait in drought studies is the leaf surface temperature that reflects the transpiration rate of the plant (as discussed above in the section Thermoimaging). A combination of shoot digital imaging, thermoimaging and automated weighing and watering to study WUE was used by Fehér-Juhász et al. [19]. These authors employed a self-constructed greenhouse-based platform for the selection of drought-tolerant transgenic wheat plants. The platform allows monitoring of the mature cereal plants' growth by multiple-view RGB imaging and assessment of the leaf surface temperature by side-view thermal camera recording the differences in temperatures of plant shoots [19]. The same platform and a similar phenotyping experimental design were used for evaluation of drought tolerance in barley. The system provides integrative analysis of plant growth and physiology, but its use for large-scale analysis is limited by a semi-automated regime requiring manual loading of the plants into the system [65].
Given that physiological responses to drought and high temperature stresses are tightly connected, similar approaches can be used to study the tolerance of plants to both drought and high temperature. The use of highthroughput phenotyping for high temperature tolerance and a description of the appropriate sensors can be found in a review by Gupta et al. [70]. More recently, the effects of the high temperature on the Arabidopsis plants were studied by Vasseur et al. [71]. The authors used commercial-prototype platform allowing the topview RGB imaging and WUE analysis followed by highly-sophisticated statistical approach to reveal contrasting adaptive strategies to the high temperature and drought stresses [71].

The salinization of soil is another phenomenon often associated with drought and high temperature stress. The example of the protocol for salt stress study in various cereals combining RGB imaging with destructive leaf sampling to measure $\mathrm{Na}^{+}$concentration was described by Berger et al. [72]. The effect of salt stress was studied by Rajendran et al. [73] using digital RGB imaging in a greenhouse-based commercial system. This study provided deep insight into the physiological processes connected with salinity in wheat. The authors used the multiple-view RGB imaging to estimate a digital area of shoot, and to visualize changes in leaf colour for quantification of the senescent area. Using non-invasive plant phenotyping and analysis of $\mathrm{Na}^{+}$concentration in 4th leaf, the authors predicted a plant salinity tolerance index that showed a good correlation with the results obtained from conventional salt-tolerance measurements [73]. Simple RGB imaging in wheat and barley was carried out in the physiological study of Harris et al. [74], and described in the methodological report of Golzarian et al. [18]. Recently, Schilling et al. applied a similar approach to select a salt-tolerant line of transgenic barley [75]. The combination of digital RGB imaging (used to measure shoot growth rate) with SLCFIM (used for the assessment of senescent areas) was used for the selection of salt-tolerant cultivars of rice by Hairmansis et al. [76]. These studies of salt-stress tolerance were performed using the same commercial platform involving SLCFIM sensor. As mentioned in the section Chlorophyll fluorescence imaging (CFIM) this type of CFIM in fact provides only estimation of a senescent area that can be obtained using an older way of estimation based on colour detection by RGB imaging. Thus, to increase the value of the physiological evaluation, the use of KCFIM is necessary for quantification of the quantum yield of photochemistry and of the other competitive processes [36].

Combination of RGB imaging, thermoimaging and TLCFIM was used in the pioneer work of Chaerle at al. who evaluated the effects of mild mottle virus infection on tobacco and bean plants [77]. The use of high- 
throughput techniques in the nutrient starving stress studies have been already reported too. The principle of the method based on RGB imaging of leaf expansion was described by Moreau et al. [78]. A comprehensive study on the phenotypic effects of nitrogen and phosphorus nutrient statuses of Brachypodium was carried out by Poire et al. employing RGB imaging to estimate growth rate [79]. A similar approach was used in a study of Neilson et al. [80] where the responses to nitrogen deficiency and drought were evaluated by RGB imaging, NIR imaging and automated weighing, respectively. The authors also developed software that extracted from the images, additive traits such as projected plant height and the height to the ligule of the youngest fully expanded leaf, which showed very good correlations with standard manually measured agronomical parameters [80]. The multiple-sensor approach was described earlier in beans by Chaerle et al., who used RGB imaging, thermoimaging and TLCFIM to evaluate the phenotypes related to magnesium deficiency and biotic stress [81]. The impact of cold stress on plant growth and physiology is routinely studied using non-invasive methods through the analysis of $\mathrm{Chl}$ fluorescence, but not using fluorescence sensors integrated into complex growth-analysing platforms [82-84]. Jansen et al. studied the effects of chilling stress in Arabidopsis and tobacco plants using a growth chamber based system equipped with digital top-view RGB screening and KCFIM [37]. Very recently an automated screening approach based on RGB imaging and KCFIM analysis for selection of pea cultivars with different cold-sensitivity was developed by Humplík et al. [20]. The reported study was not intended only for selection of cold-sensitive/tolerant varieties of pea but also for studies of plant cold-response strategies in general. Since the CFIM analysis is not limited to plant morphology and the image analysis was sensitive enough to detect tiny tendrils of pea, the described procedure should be theoretically employed for shoot analyses of other plant species [20].

\section{Conclusions}

This mini-review focuses on recent advances towards development of integrative automated platforms for high-throughput plant phenotyping that employ multiple sensors for simultaneous analysis of plant shoots. In both basic and applied science, the recently emerging approaches have found importance as tools in unravelling complex questions of plant growth, development, responses to environment, as well as selection of appropriate genotypes in molecular breeding strategies. As far as phenotype is an interactive network of responses by the plant to its environment that affects in turn, the expression of the genotype it is worth pointing out that attention to the way the analyses are done, under precisely controlled conditions allowing for direct linking the huge amount of complex phenotyping data obtained to the particular conditions. It would also help the end user - the biologist - to narrow his/her view on the importance of various parameters and indices available from the specialized measurements (specifically CFIN and reflectance measurements) and evaluate which of them provide the required information in the best way and hence thus the most suitable for high-throughput plant phenotyping. Such information and standardized protocols applicable for the particular phenotyping methodologies should be available in the near future due to the phenotyping community efforts.

\begin{abstract}
Abbreviations
Chl: Chlorophyll; CFIM: Chlorophyll fluorescence imaging; CFIN: Chlorophyll fluorescence induction; DW: Dry weight; $F_{\mathrm{M}}$ : Maximal chlorophyll

fluorescence levels for dark- adapted state; FW: Fresh weight; $F_{V}$ : variable chlorophyll fluorescence level for a dark-adapted state; $\Phi_{\text {po: }}$ The maximal quantum yield of photosystem II photochemistry for a dark-adapted state; KCFIM: Kinetic chlorophyll fluorescence imaging; NDVI: Normalized difference vegetation index; PAM: Pulse amplitude modulation; PRI: Photochemical reflectance index; PSII: Photosystem II; RGB: Red-green-blue; SLCFIM: Singlelevel chlorophyll fluorescence imaging; SP: Saturation pulse; SWIR: Short wavelength infrared; TLCFIM: Two-level chlorophyll fluorescence imaging; VIS-NIR: Visible-near infrared; WUE: Water-use efficiency.
\end{abstract}

\section{Competing interests}

The authors declare that they have no competing interests.

\section{Authors' contributions}

JFH, DL, AH and LS drafted the manuscript. All authors read and approved the final manuscript.

\section{Acknowledgment}

This work was supported by the grant No LO1204 (Sustainable development of research in the Centre of the Region Haná) from the National Program of Sustainability I, Ministry of Education, Youth and Sports, Czech Republic.

\section{Author details}

${ }^{1}$ Department of Chemical Biology and Genetics, Centre of the Region Haná for Biotechnological and Agricultural Research, Faculty of Science, Palacký University, Šlechtitelů 11, Olomouc CZ-78371, Czech Republic. ²Department of Biophysics, Centre of the Region Haná for Biotechnological and Agricultural Research, Faculty of Science, Palacký University, Šlechtitelů 11, Olomouc CZ-78371, Czech Republic.

Received: 5 February 2015 Accepted: 9 April 2015

Published online: 17 April 2015

\section{References}

1. Araus JL, Cairns JE. Field high-throughput phenotyping: the new crop breeding frontier. Trends Plant Sci. 2014;19:52-61.

2. Granier C, Vile D. Phenotyping and beyond: modelling the relationships between traits. Curr Opin Plant Biol. 2014;18:96-102.

3. Fiorani F, Schurr U. Future scenarios for plant phenotyping. Annu Rev Plant Biol. 2013:64:267-91.

4. Fiorani F, Rascher U, Jahne S, Schurr U. Imaging plants dynamics in heterogenic environments. Curr Opin Biotech. 2012;23:227-35.

5. Walter A, Studer B, Kölliker R. Advanced phenotyping offers opportunities for improved breeding of forage and turf species. Annals Bot. 2012;110:1271-9.

6. Jansen M, Pinto F, Nagel KA, van Dusschoten D, Fiorani F, Rascher U, et al. Non-invasive phenotyping methodologies enable the accurate characterization of growth and performance of shoots and roots. In: Tuberosa R, Graner A, Frison E, editors. Genomics of Plant Genetic Resources. Volume 1st ed. Netherlands: Springer; 2014. p. 173-206. 
7. Pieruschka R, Poorter H. Phenotyping plants: genes, phenes and machines. Funct Plant Biol. 2012;39:813-20.

8. Roberts MJ, Long SP, Tieszen LL, Beadle CL. Measurement of plant biomass and net primary production of herbaceous vegetation. In: Hall DO, Scurlock JMO, Bolhar-Nordenkampf HR, Leegood RC, Long SP, editors. Photosynthesis and Production in a Changing Environment. Netherlands: Springer; 1993.

9. Rouphael $Y$, Mouneimne AH, Ismail A, Mendoza-De Gyves E, Rivera CM, Colla G. Modeling individual leaf area of rose (Rosa hybrida L.) based on leaf length and width measurement. Photosynthetica. 2010;48:9-15.

10. Cemek B, Unlukara A, Kurunc A. Nondestructive leaf-area estimation and validation for green pepper (Capsicum annuum L.) grown under different stress conditions. Photosynthetica. 2011;49:98-106.

11. Misle E, Kahlaoui B, Hachicha M, Alvarado P. Leaf area estimation in muskmelon by allometry. Photosynthetica. 2013;51:613-20.

12. Green JM, Appel H, MacNeal Rehrig E, Harnsomburana J, Chang J-F, Balint-Kurti $P$, et al. PhenoPhyte: a flexible affordable method to quantify $2 \mathrm{D}$ phenotypes from imagery. Plant Methods. 2012;8:45.

13. Zhang X, Hause RJ, Borevitz JO. Natural genetic variation for growth and development revealed by high-throughput phenotyping in Arabidopsis thaliana. G3-Genes Genom Genet. 2012;2:29-34.

14. Tessmer OL, Jiao Y, Cruz JA, Kramer DM, Chen J. Functional approach to high-throughput plant growth analysis. BMC Syst Biol. 2013;7 Suppl 6:S17.

15. Furbank RT, Tester M. Phenomics-technologies to relieve the phenotyping bottleneck. Trends Plant Sci. 2011;16:635-44.

16. Honsdorf N, March TJ, Berger B, Tester M, Pillen K. High-throughput phenotyping to detect drought tolerance QTL in wild barley introgression lines. PLoS One. 2014;9, e97047.

17. Walter A, Scharr H, Gilmer F, Zierer R, Nagel KA, Ernst M, et al. Dynamics of seedling growth acclimation towards altered light conditions can be quantified via GROWSCREEN: a setup and procedure designed for rapid optical phenotyping of different plant species. New Phytol. 2007;174:447-55.

18. Golzarian MR, Frick RA, Rajendran K, Berger B, Roy S, Tester M, et al. Accurate inference of shoot biomass from high-throughput images of cereal plants. Plant Methods. 2011;7:1-11.

19. Fehér-Juhász E, Majer P, Sass L, Lantos C, Csiszár J, Turóczy Z, et al. Phenotyping shows improved physiological traits and seed yield of transgenic wheat plants expressing the alfalfa aldose reductase under permanent drought stress. Acta Physiol Plant. 2014;36:663-73.

20. Humplík JF, Lazár D, Fürst T, Husičková A, Hýbl M, Spíchal L. Automated integrative high-throughput phenotyping of plant shoots: a case study of the cold-tolerance of pea (Pisum sativum L.). Plant Methods. 2015;11:1-11.

21. Berger B, de Regt B, Tester M. High-throughput phenotyping of plant shoots. In: Normanly J, editor. High-Throughput Phenotyping in Plants. New York City: Humana Press; 2012. p. 9-20.

22. Camargo A, Papadopoulou D, Spyropoulou Z, Vlachonasios K, Doonan JH, Gay AP. Objective definition of Rosette shape variation using a combined computer vision and data mining approach. PLoS One. 2014;9, e96889.

23. Crowell S, Falcão AX, Shah A, Wilson Z, Greenberg AJ, McCouch SR. High-resolution inflorescence phenotyping using a novel image-analysis pipeline, PANorama. Plant Physiol. 2014;165:479-95.

24. Paulus S, Dupuis J, Riedel S, Kuhlmann H. Automated analysis of barley organs using 3d laser scanning: an approach for high throughput phenotyping. Sensors. 2014;14:12670-86.

25. Fanourakis D, Briese C, Max JF, Kleinen S, Putz A, Fiorani F, et al. Rapid determination of leaf area and plant height by using light curtain arrays in four species with contrasting shoot architecture. Plant Methods. 2014;10:9.

26. Neumann K, Klukas C, Friedel S, Rischbeck P, Chen D, Entzian A, Stein N, Graner A, Kilian B. Dissecting spatio-temporal biomass accumulation in barley under different water regimes using high-throughput image analysis. Plant Cell Environ 2015. doi: 10.1111/pce.12516, in press.

27. Kautsky H, Hirsch A. Neue Versuche zur Kohlensureassimilation. Naturwissenschaften. 1931:19:964.

28. Lazár D. Chlorophyll a fluorescence induction. Biochim Biophys Acta. 1999:1412:1-28.

29. Lazár D. The polyphasic chlorophyll a fluorescence rise measured under high intensity of exciting light. Funct Plant Biol. 2006;33:9-30.

30. Schreiber U, Schliwa U, Bilger W. Continuous recording of photochemical and non-photochemical chlorophyll fluorescence quenching with a new type of modulation fluorometer. Photosynth Res. 1986;10:51-62.
31. Omasa K, Shimazaki K-I, Aiga I, Larcher W, Onoe M. Image analysis of chlorophyll fluorescence transients for diagnosing the photosynthetic system of attached leaves. Plant Physiol. 1987;84:748-52.

32. Daley PF, Raschke K, Ball JT, Berry JA. Topography of photosynthetic activity of leaves obtained from video images of chlorophyll fluorescence. Plant Physiol. 1989;90:1233-8.

33. Lazár D, Sušila P, Nauš J. Early detection of plant stress from changes in distributions of chlorophyll a fluorescence parameters measured with fluorescence imaging. J Fluoresc. 2006;16:173-6.

34. Gorbe E, Calatayud A. Applications of chlorophyll fluorescence imaging technique in horticultural research: A review. Sci Hortic. 2012;138:24-35.

35. Harbinson J, Prinzenberg AE, Kruijer W, Aarts MGM. High throughput screening with chlorophyll fluorescence imaging and its use in crop improvement. Curr Opin Biotech. 2012;23:221-6.

36. Lazár D. Parameters of photosynthetic energy partitioning. J Plant Physiol. 2015;175:131-47.

37. Jansen M, Gilmer F, Biskup B, Nagel KA, Rascher U, Fischbach A, et al. Simultaneous phenotyping of leaf growth and chlorophyll fluorescence via GROWSCREEN FLUORO allows detection of stress tolerance in Arabidopsis thaliana and other rosette plants. Funct Plant Biol. 2009;36:902-14.

38. Hashimoto Y, Ino T, Kamer PJ, Naylor AW, Strain BR. Dynamic analysis of water stress of sunflower leaves by means of a thermal image processing system. Plant Physiol. 1984;76:266-9.

39. Kaňa R, Vass I. Thermoimaging as a tool for studying light-induced heating of leaves Correlation of heat dissipation with the efficiency of photosystem II photochemistry and non-photochemical quenching. Environ Exp Bot. 2008;64:90-6.

40. Siddiqui ZS, Cho I-L, Park S-H, Kwon T-R, Ahn B-O, Lee G-S, et al. Phenotyping of rice in salt stress environment using high-throughput infrared imaging. Acta Bot Croat. 2014;73:149-58.

41. Virlet N, Lebourgeois V, Martinez S, Costes E, Labbé S, Regnard J-L. Stress indicators based on airborne thermal imagery for field phenotyping a heterogeneous tree population for response to water constraints. J Exp Bot. 2014;65:5429-42

42. Jones HG, Serraj R, Loveys BR, Xiong L, Wheaton A, Price AH. Thermal infrared imaging of crop canopies for the remote diagnosis and quantification of plant responses to water stress in the field. Funct Plant Biol. 2009;36:978-89.

43. Costa JM, Ortuño MF, Lopes CM, Chaves MM. Grapevine varieties exhibiting differences in stomatal response to water deficit. Funct Plant Biol. 2012;39:179-89.

44. Grant OM, Davies MJ, James CM, Johnson AW, Leinonen I, Simpson DW. Thermal imaging and carbon isotope composition indicate variation amongst strawberry (Fragaria $\times$ ananassa) cultivars in stomatal conductance and water use efficiency. Environ Exp Bot. 2012;76:7-15.

45. Costa JM, Grant OM, Chaves MM. Thermography to explore plantenvironment interactions. J Exp Bot. 2013;64:3937-49.

46. Rouse JWJ, Haas RH, Schell JA, Deering DW. Monitoring vegetation systems in the Great Plains with ERTS. In: Freden SC, Marcanti EP, Becker MA, editors. NASA SP-351. Proceedings of the 3rd Earth Resources Technology Satellite1Symposium. Washington DC: NASA Scientific and Technical Information Office; 1974. p. 309-17.

47. Gamon JA, Peñuelas J, Field CB. A narrow-waveband spectral index that tracks diurnal changes in photosynthetic efficiency. Remote Sens Environ. 1992:41:35-44.

48. Carter GA. Primary and secondary effects of water content on the spectra reflectance of leaves. Am J Bot. 1991;78:916-24.

49. Huber S, Tagesson T, Fensholt R. An automated field spectrometer system for studying VIS, NIR and SWIR anisotropy for semi-arid savanna. Remote Sens Environ. 2014:152:547-56.

50. Lamb DW, Schneider DA, Stanley JN. Combination active optical and passive thermal infrared sensor for low-level airborne crop sensing. Precis Agric. 2014;15:523-31.

51. Saberioon MM, Amin MSM, Anuar AR, Gholizadeh A, Wayayok A Khairunniza-Bejo S. Assessment of rice leaf chlorophyll content using visible bands at different growth stages at both the leaf and canopy scale. Int J Appl Earth Observ Geoinform. 2014;32:35-45.

52. Garriga M, Retamales JB, Romero-Bravo S, Caligari PDS, Lobos GA Chlorophyll, anthocyanin, and gas exchange changes assessed by spectroradiometry in Fragaria chiloensis under salt stress. J Integr Plant Biol. 2014;56:505-15. 
53. Mahajan GR, Sahoo RN, Pandey RN, Gupta VK, Kumar D. Using hyperspectral remote sensing techniques to monitor nitrogen, phosphorus, sulphur and potassium in wheat (Triticum aestivum L.). Precis Agric. 2014;15:499-522.

54. Petach AR, Toomey M, Aubrecht DM, Richardson AD. Monitoring vegetation phenology using an infrared-enabled security camera. Agri Forest Meteorol. 2014;195-196:143-51.

55. Ollinger SV. Sources of variability in canopy reflectance and the convergent properties of plants. New Phytol. 2011;189:375-94.

56. Mir RR, Zaman-Allah M, Sreenivasulu N, Trethowan R, Varshney RK. Integrated genomics, physiology and breeding approaches for improving drought tolerance in crops. Theor Appl Genet. 2012;125:625-45.

57. Berger B, Parent B, Tester M. High-throughput shoot imaging to study drought responses. J Exp Bot. 2010;61:3519-28.

58. Granier C, Aguirrezabal L, Chenu K, Cookson SJ, Dauzat M, Hamard P, et al. PHENOPSIS, an automated platform for reproducible phenotyping of plant responses to soil water de cit in. New Phytol. 2006;169:623-35.

59. Skirycz A, Vandenbroucke K, Clauw P, Maleux K, De Meyer B, Dhondt S, et al. Survival and growth of Arabidopsis plants given limited water are not equal. Nat Biotechnol. 2011;29:212-4.

60. Clauw P, Coppens F, De Beuf K, Dhondt S, Van Daele T, Maleux K, et al. Leaf Responses to Mild Drought Stress in Natural Variants of Arabidopsis thaliana. Plant Physiol. 2015;114:254-84.

61. Tisné $S$, Serrand $Y$, Bach $L$, Gilbault $E$, Ben Ameur $R$, Balasse $H$, et al. Phenoscope: An automated large-scale phenotyping platform offering high spatial homogeneity. Plant J. 2013;74:534-44.

62. Pereyra-Irujo GA, Gasco ED, Peirone LS, Aguirrezábal LA. GlyPh: a low-cost platform for phenotyping plant growth and water use. Funct Plant B iol. 2012;39:905-13.

63. Coupel-Ledru A, Lebon É, Christophe A, Doligez A, Cabrera-Bosquet L, Péchier $P$, et al. Genetic variation in a grapevine progeny (Vitis vinifera L. Cvs Grenache $\times$ Syrah) reveals inconsistencies between maintenance of daytime leaf water potential and response of transpiration rate under drought. J Exp Bot. 2014;65:6205-218.

64. Chen D, Neumann K, Friedel S, Kilian B, Chen M, Altmann T, et al. Dissecting the Phenotypic Components of Crop Plant Growth and Drought Responses Based on High-Throughput Image Analysis. Plant Cell. 2014;26:4636-55.

65. Cseri A, Sass L, Törjék O, Pauk J, Vass I, Dudits D. Monitoring drought responses of barley genotypes with semi-robotic phenotyping platform and association analysis between recorded traits and allelic variants of some stress genes. Aust J Crop Sci. 2013:7:1560-70.

66. Petrozza A, Santaniello A, Summerer S, Di Tommaso G, Di Tommaso D, Paparelli E, et al. Physiological responses to Megafol ${ }^{\oplus}$ treatments in tomato plants under drought stress: A phenomic and molecular approach. Sci Hortic (Amsterdam). 2014;174:185-92.

67. Harshavardhan VT, Van Son L, Seiler C, Junker A, Weigelt-Fischer K, Klukas C, et al. AtRD22 and AtUSPL1, Members of the Plant-Specific BURP Domain Family Involved in Arabidopsis thaliana Drought Tolerance. PLoS One. 2014;9, e110065

68. Bresson J, Varoquaux F, Bontpart T, Touraine B, Vile D. The PGPR strain Phyllobacterium brassicacearum STM196 induces a reproductive delay and physiological changes that result in improved drought tolerance in Arabidopsis. New Phytol. 2013;200:558-69.

69. Bresson J, Vasseur F, Dauzat M, Labadie M, Varoquaux F, Touraine B, et al. Interact to survive: Phyllobacterium brassicacearum improves Arabidopsis tolerance to severe water deficit and growth recovery. PLoS One. 2014;9, e107607.

70. Gupta PK, Balyan HS, Gahlaut V, Kulwal PL. Phenotyping, genetic dissection, and breeding for drought and heat tolerance in common wheat: status and prospects. Plant Breeding Reviews. 2012;36:85-147.

71. Vasseur F, Bontpart T, Dauzat M, Granier C, Vile D. Multivariate genetic analysis of plant responses to water deficit and high temperature revealed contrasting adaptive strategies. J Exp Bot. 2014;65:6457-69.

72. Berger B, de Regt B, Tester M. Trait dissection of salinity tolerance with plant phenomics. In: Shabala S, Cuin TA, editors. Plant Salt Tolerance. New York City: Humana Press; 2012. p. 399-413.

73. Rajendran K, Tester M, Roy SJ. Quantifying the three main components of salinity tolerance in cereals. Plant, Cell Environ. 2009;32:237-49.

74. Harris BN, Sadras VO, Tester M. A water-centred framework to assess the effects of salinity on the growth and yield of wheat and barley. Plant Soil. 2010;336:377-89.
75. Schilling RK, Marschner P, Shavrukov Y, Berger B, Tester M, Roy SJ, et al. Expression of the Arabidopsis vacuolar $\mathrm{H}^{+}$-pyrophosphatase gene (AVP1) improves the shoot biomass of transgenic barley and increases grain yield in a saline field. Plant Biotechnol J. 2014;12:378-86.

76. Hairmansis A, Berger B, Tester M, Roy SJ. Image-based phenotyping for nondestructive screening of different salinity tolerance traits in rice. Rice. 2014;7:16.

77. Chaerle L, Pineda M, Romero-Aranda R, Van Der Straeten D, Barón M. Robotized thermal and chlorophyll fluorescence imaging of pepper mild mottle virus infection in Nicotiana benthamiana. Plant Cell Physiol. 2006;47:1323-36

78. Moreau D, Schneider C, Huguet T, Salon C, Munier-Jolain N. Can differences of nitrogen nutrition level among Medicago truncatula genotypes be assessed non-destructively? Probing with a recombinant inbred lines population. Plant Signal Behav. 2009:4:30-2.

79. Poiré R, Chochois V, Sirault XRR, Vogel JP, Watt M, Furbank RT. Digital imaging approaches for phenotyping whole plant nitrogen and phosphorus response in Brachypodium distachyon. J Integr Plant Biol. 2014;56:781-96.

80. Neilson EH, Edwards AM, Blomstedt CK, Berger B, Moller BL, Gleadow RM. Utilization of a high-throughput shoot imaging system to examine the dynamic phenotypic responses of a C 4 cereal crop plant to nitrogen and water deficiency over time. J Exp Bot. 2015;66:1817-32

81. Chaerle L, Hagenbeek D, Vanrobaeys X, Van Der Straeten D. Early detection of nutrient and biotic stress in Phaseolus vulgaris. Int J Remot Sens. 2007;28:3479-92

82. Devacht S, Lootens P, Baert J, Van Waes J, Van Bockstaele E, Roldán-Ruiz I. Evaluation of cold stress of young industrial chicory (Cichorium intybus L.) plants by chlorophyll a fluorescence imaging. I. Light induction curve. Photosynthetica. 2011;49:161-71.

83. Lootens P, Devacht S, Baert J, Van Waes J, Van Bockstaele E, Roldán-Ruiz I. Evaluation of cold stress of young industrial chicory (Cichorium intybus L.) by chlorophyll a fluorescence imaging. II. Dark relaxation kinetics. Photosynthetica. 2011;49:185-94.

84. Mishra A, Mishra KB, Höermiller II, Heyer AG, Nedbal L. Chlorophyll fluorescence emission as a reporter on cold tolerance in Arabidopsis thaliana accessions. Plant Signal Behav. 2011;6:301.

\section{Submit your next manuscript to BioMed Central and take full advantage of:}

- Convenient online submission

- Thorough peer review

- No space constraints or color figure charges

- Immediate publication on acceptance

- Inclusion in PubMed, CAS, Scopus and Google Scholar

- Research which is freely available for redistribution 